\title{
Aplicación de sensores piezoeléctricos cerámicos a la caracterización biomecánica
}

\author{
J.C. MORENO', J.F. FERNÁNDEZ'², P. OCHOA², R.CERES', L. CALDERÓN'1, E. ROCON, J.L. PONS'1 \\ 'Instituto de Automática Industrial, CSIC, Ctra. Campo Real, km. 0,200, 28500 Arganda del Rey, Madrid \\ ${ }^{2}$ Instituto de Cerámica y Vidrio, CSIC, Camino de Valdelatas s/n, 28049 Madrid
}

\begin{abstract}
El presente trabajo analiza las aplicaciones potenciales de los sensores piezoeléctricos cerámicos para la medición de variables cinemáticas y cinéticas en las áreas de biomedicina y biomecánica. A partir del análisis teórico del sensor se realiza un análisis armónico por elementos finitos del mismo, que muestra la existencia de gradientes de potencial entre los pares de electrodos. Se propone un sensor triaxial piezoeléctrico para la medida concomitante de esfuerzos durante el contacto pie-suelo en el ciclo de marcha humana.

Palabras clave: Sensor de presión, medida triaxial de fuerza, piezoeléctricos cerámicos.

Ceramic Piezoelectric Sensors: Application to Biomecanic Characterization

This work analyzed the potential applications of ceramic piezoelectrics as sensors of kinematic and kinetic parameters in the field of the biomechanics and biomedicine. On the basis of a theoretically analysis of the sensor a Finite Element Analysis of the harmonic shown the appearance of field gradients between the electrodes. A piezoelectric sensor is proposed as triaxial force transducer for plantar pressure measurement during human walking.
\end{abstract}

Keywords: Pressure sensor, triaxial force measurement, ceramic piezoelectrics.

\section{INTRODUCCIÓN}

Los fenómenos físicos que puedan dar lugar a la obtención de una deformación mecánica son susceptibles de ser medidos mediante el empleo de sensores piezoeléctricos. En efecto, en la actualidad y en número creciente, son comunes las aplicaciones en las que variables tales como la aceleración [1], fuerza, presión (y variables derivadas como campo acústico o campo ultrasónico [2]) se determinan en entornos industriales mediante sensores piezoeléctricos.

Las nuevas tecnologías de medida y actuación están paulatinamente alcanzando otros ámbitos de aplicación además de las meramente industriales [3]. Un ejemplo de esta tendencia es el empleo de nuevos sensores y actuadores electrocerámicos en aplicaciones biomédicas y biomecánicas. Recientemente, se ha propuesto el empleo de motores piezoeléctricos para el accionamiento de prótesis avanzadas de miembro superior [4].

Las aplicaciones biomédicas y en particular las biomecánicas requieren la determinación de variables tanto cinématicas (posición, velocidad o aceleración de distintos segmentos del cuerpo humano) como cinéticas. Entre estas últimas cabe destacar aquellas que requieren la caracterización del par y la potencia desarrollada durante la marcha, o la distribución de presiones entre elementos protésicos y el cuerpo humano. El desarrollo de aplicaciones en este campo está claramente limitado por la autonomía de las soluciones propuestas: cualquier aplicación autónoma requiere elementos sensores que puedan operar como elementos miniaturizados y compactos, y así ser integrables en sistemas portatiles.

En este sentido existen ejemplos de sensores triaxiales de esfuerzo para ser integrados en calzado, [5], y obtener así una estimación de la presión plantar. También se han reportado desarrollos de sistemas portátiles para la monitorización triaxial de la condición de contacto entre el encaje de la prótesis y el miembro [6].

El presente trabajo, a partir de una revisión de las posibles aplica- ciones de los sensores piezoeléctricos en el área de la biomecánica, se centra en el estudio de un sensor triaxial de fuerza fundamentado en la combinación de esfuerzos sobre el elemento piezocerámico prismático. Mediante la técnica de Análisis por Elementos Finitos se modeliza el efecto de los electrodos en las seis caras de la cerámica sobre la sensibilidad a cada componente de la fuerza. Se plantea el empleo de este tipo de sensores para la determinación de la distribución de la presión plantar y el cálculo del vector de fuerza de reacción entre el pie y el suelo durante la fase de apoyo.

\section{LA MARCHA HUMANA: CARACTERIZACIÓN CINÉ- TICA Y CINEMÁTICA}

El estudio biomecánico de la locomoción humana, considerada como un patrón periódico característico que se conoce como ciclo de marcha, se divide en dos fases según la situación del miembro inferior con respecto al suelo: la fase de apoyo y la fase de oscilación. Durante la fase de apoyo, de acuerdo con la tercera ley del movimiento de Newton, la interacción entre el miembro y el suelo genera una fuerza de reacción igual en magnitud y opuesta en dirección a la fuerza que el cuerpo ejerce sobre la superficie de contacto.

El ciclo de marcha humana está entonces caracterizado por la variación temporal de las variables cinemáticas del miembro inferior: ángulo de la rodilla y del tobillo; así como por la variación temporal de las variables cinéticas: par de la rodilla y del tobillo y de la fuerza de reacción, [7]. La figura 1 muestra la evolución de estas variables (para el caso de las variables asociadas a la rodilla) en función del porcentaje del ciclo de marcha, entendiendo este como el intervalo entre dos contactos consecutivos del mismo pie sobre el suelo.

La fuerza de reacción que ejerce el suelo tiene componentes en el 
plano frontal, sagital y transversal (figura 2), combinando los efectos gravitatorios y dinámicos del cuerpo. Así, el vector de la fuerza de reacción constituye un parámetro cinético de alta relevancia en el análisis de la marcha ya que al actuar sobre cada una de las articulaciones del miembro inferior produce momentos y genera movimientos. Un conocimiento de dicho vector ayudará a entender sus efectos sobre el cuerpo y a predecir la actividad muscular. Otra utilidad importante de conocer dicho vector de fuerza se encuentra en el diseño y prescripción de aparatos ortopédicos tales como prótesis y ortesis del miembro inferior, así como la aportación de criterios importantes para el funcionamiento, seguridad y estabilidad dinámica de las articulaciones con este tipo de ayudas. La figura 3 muestra la evolución antero-posterior del vector de fuerza de reacción en tres instantes distintos y consecutivos de la fase de apoyo.

Por otra parte, la transferencia del peso del cuerpo también determina una distribución de presiones en la planta del pie en función de la fuerza de reacción y de la superficie de contacto. La distribución de presiones plantares refleja la interacción mecánica del pie con el suelo y permite evaluar su función durante la locomoción.

Por esto se han desarrollado diversas técnicas cuantitativas para obtener medidas de cargas presentes en los procesos de locomoción, las cuales están basadas en diferentes tipos de transductores que proporcionan una señal eléctrica en función de la fuerza o presión aplicada. Para la medida de la fuerza de reacción que ejerce el suelo se utilizan principalmente sistemas de plataformas dinamométricas de uso extendido en los laboratorios de análisis de la marcha. Estos sistemas emplean principalmente transductores piezoeléctricos o extensométricos, distribuidos sobre una plataforma que se encuentra fija en el suelo. Normalmente las plataformas incorporan cuatro sensores triaxiales, que miden la fuerza que actúa entre el pie y el suelo en tres ejes (caso tridimensional): transversal, anteroposterior y vertical. Una técnica utilizada en las plataformas que permite la medida de las tres componentes consiste en separar la sensibilidad a cargas verticales y a esfuerzos horizontales por grupos de captadores especializados.
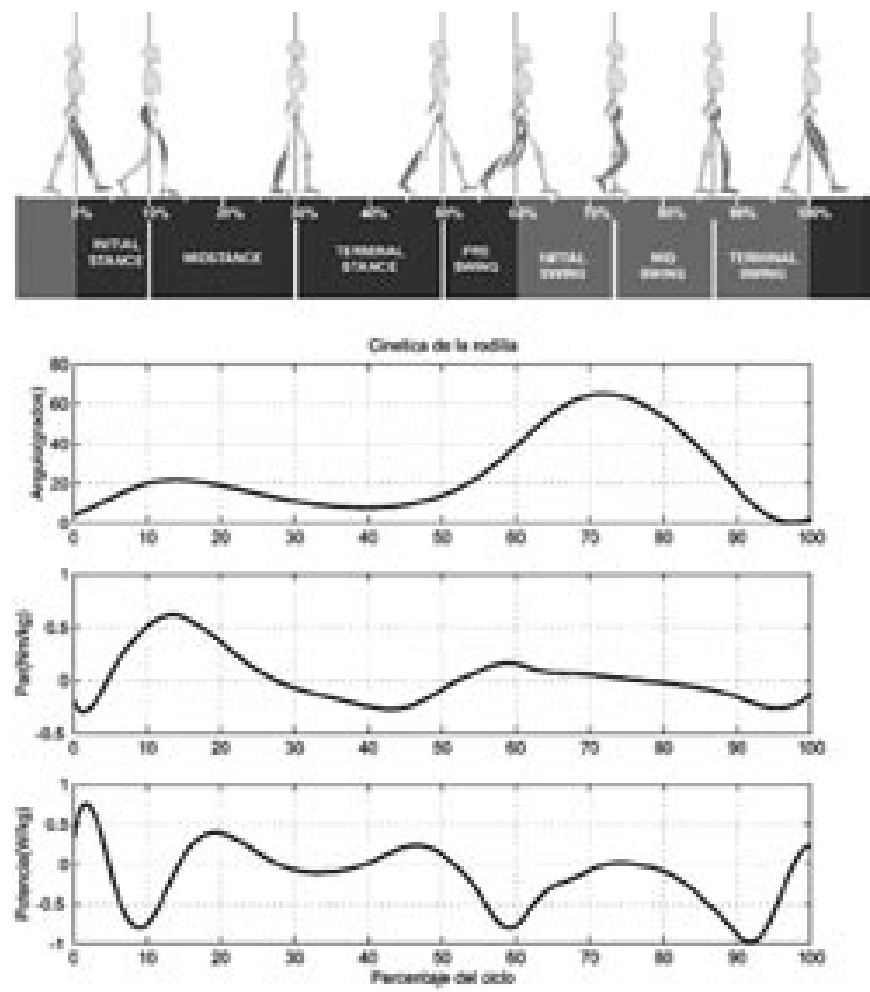

Figura 1. Caracterización del ciclo de marcha mediante variables cinemáticas (ángulo) y cinéticas (par y potencia) a nivel de la rodilla.

\section{MEDIDA DE FUERZA DE REACCIÓN: PLANTILLAS PIEZOELÉCTRICAS}

El método cuantitativo disponible para la medida de presiones plantares lo constituye un sistema de sensores que registran las presiones que se produzcan entre el pie y el calzado. Estos sensores se distribuyen en una plantilla, y así permiten un seguimiento continuo de la interacción del pie del sujeto con el suelo sin las restricciones de movilidad en el espacio que presentan las plataformas. En la figura 4 se muestra la distribución de presión plantar durante la fase de apoyo en un ciclo normal de marcha. Un sensor discreto o una matriz de sensores se utilizan para medir la fuerza que actúa mientras el pie está en contacto con la superficie de soporte. La magnitud de la presión se parametriza entre el área de contacto conocida del sensor o los sensores involucrados durante el contacto, permitiendo la determinación de las fuerzas involucradas.

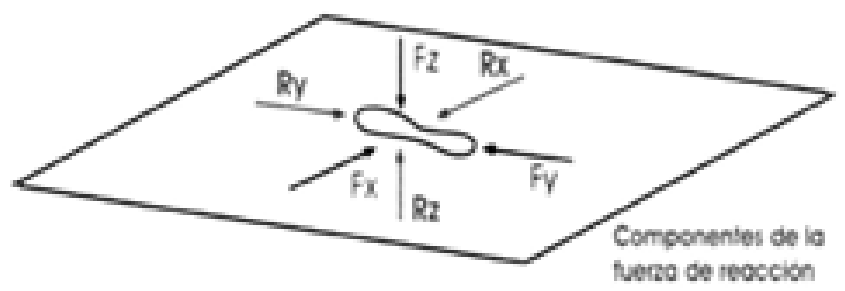

Figura 2. Componentes de la fuerza aplicada por el cuerpo y de la fuerza de reacción del suelo.

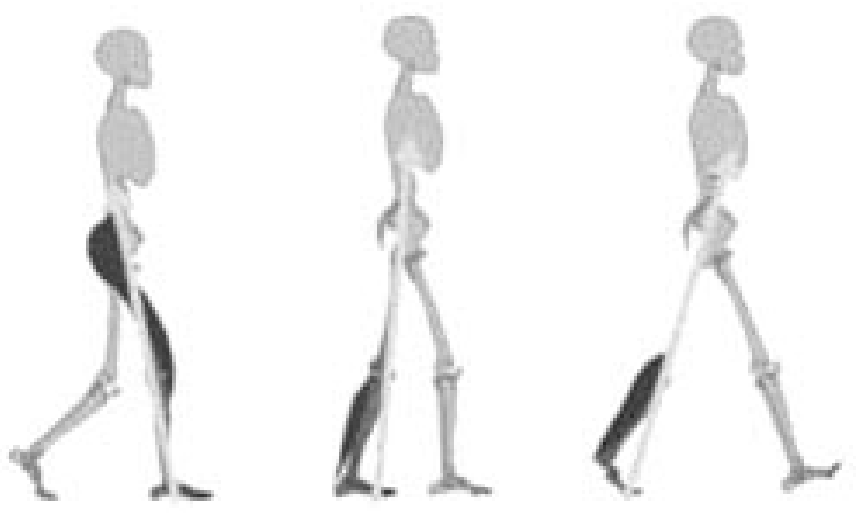

Figura 3. Evolución de la componente antero-posterior del vector de fuerza de reacción del suelo (VFRS), durante la fase de apoyo.

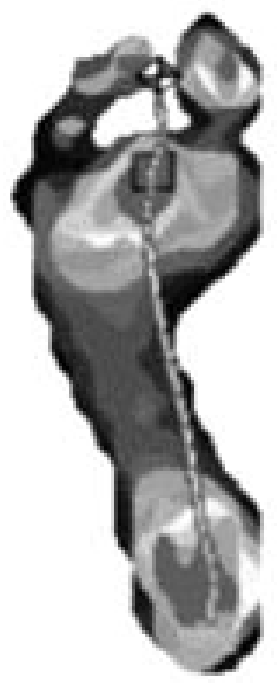

Figura 4. Distribución de la presión plantar en un instante de la fase de apoyo. 


\subsection{Estado del arte}

La determinación de las presiones plantares pueden realizarse mediante una gran variedad de técnicas que incluyen resistencias sensoras de fuerzas, hidroceldas, microcápsulas, transductores capacitivos, cerámicas piezoeléctricas y láser. Los transductores capacitivos y resistivos son los dos tipos básicos en uso hoy en día para la medida de presión plantar. Los sistemas de registro de presión plantar pueden clasificarse en sistemas:

- Discretos. Se utilizan transductores individuales localizados en puntos anatómicos específicos en la superficie plantar.

- Matriciales. Usan una matriz de sensores organizados en filas y columnas. Así, las medidas pueden evaluar la distribución de presiones que actúan sobre toda la superficie plantar del pie simultáneamente.

En el desarrollo de sistemas para el estudio de presiones plantares principalmente se han explotado las siguientes tecnologías, [8-12]:

- Transductores capacitivos. Consisten en dos placas que almacenan una carga eléctrica. Cuando una fuerza se aplica, la distancia entre las placas se reduce y la capacitancia aumenta. La desventaja de utilizar transductores capacitivos es que la plantilla debe ser más gruesa (aprox. $2 \mathrm{~mm}$ ) en comparación con otros sensores.

- Resistencias sensoras de fuerzas (RSF). Son dispositivos laminares con patrones de metal impresos en un polímero conductor. $\mathrm{Al}$ aumentar la presión, la lámina conductora ve disminuida su resistencia eléctrica. La señal de salida de este dispositivo puede ser fuerza o presión. Las limitaciones en el empleo de estos sensores radican en que sólo informan de la componente vertical.

- Hidroceldas. Una hidrocelda consiste en un sensor discreto piezoresistivo contenido en una celda llena de fluido. Las hidroceldas se embeben en la plantilla. Cuando se aplica una carga a la hidrocelda, la fuerza aplicada causa un incremento de su resistencia. El sensor piezoresistivo de la celda genera una carga eléctrica como resultado del incremento de su resistencia. Las cualidades de estos sensores basados en elementos piezoresistivo permiten que hayan sido propuestos para evaluar tanto las fuerzas de corte como las fuerzas axiales. No obstante es necesaria una investigación más exhaustiva para confirmar la fiabilidad de dichas cualidades.

- Cerámicas Piezoeléctricas. El principio básico de operación consiste en que al aplicar una fuerza que comprime el material cerámico se produce una tensión eléctrica. Como ejemplo, el sistema BioFoot del Instituo de Biomecánica de Valencia, utiliza 64 cerámicas piezoeléctricas de reducido tamaño, distribuidas selectivamente y concentradas en las zonas de interés.

Las consideraciones importantes que se deben tener en cuenta en el diseño de este tipo de sistemas son la resolución, la frecuencia de muestreo, la fiabilidad y la calibración de los sensores a emplear. La resolución está relacionada, para un determinado tipo de sensores, tanto con el tamaño como con el número de los mismos. En general, un sistema tendrá una mayor resolución cuánto mayor sea el número de sensores que utiliza. Las diferencias en el tamaño de los elementos sensores pueden alterar la lectura de la presión, al ser ésta una función tanto de la fuerza como del área. Es decir, la fuerza aplicada a un sensor de gran tamaño no proporcionará la misma lectura de presión que la aplicada a uno pequeño. Por tanto, la dimensionalidad del sensor será también un factor importante.

Como aspecto común a los diferentes sensores presentados, cabe resaltar que todos ellos aportan únicamente la componente vertical de la fuerza de reacción. Es por tanto interesante el desarrollo de sensores compactos que aporten información sobre las tres componentes de la reacción entre el pie y el suelo, de forma dinámica durante el ciclo de marcha. La tabla I presenta una comparación de diferentes tipos de sensores para la medida de la presión plantar, así como sus características más relevantes.

\section{TABLA I: COMPARACIÓN DE SISTEMAS DE MEDIDA DE PRESIÓN PLANTAR}

\begin{tabular}{|c|c|c|c|c|c|c|c|c|}
\hline Fabricante & $\begin{array}{l}\text { Tipo de } \\
\text { Sensor }\end{array}$ & $\begin{array}{c}\text { Espesor } \\
(\mathrm{mm})\end{array}$ & Tamaños & $\begin{array}{l}\mathrm{N}^{\circ} \text {. de } \\
\text { sensores }\end{array}$ & Frecuencia & $\begin{array}{l}\text { Rango de } \\
\text { Medida }\end{array}$ & Resolución & Otras caraterísticas \\
\hline $\begin{array}{l}\text { Novel } \\
\text { Electronics } \\
\text { (Pedar } \\
\text { Insoles) }\end{array}$ & Capacitivo & 2.4 & $\begin{array}{l}22-47 \\
\text { europeo }\end{array}$ & $256 \max$ & $100 \mathrm{~Hz}$ & $2-60 \mathrm{~N} / \mathrm{cm}^{2}$ & $1 \mathrm{~N} / \mathrm{cm}^{2}$ & $\begin{array}{l}\text { Sistema de } \\
\text { almacemamiento } \\
\text { móvil. hasta } 60 \\
\text { mins. }\end{array}$ \\
\hline $\begin{array}{l}\text { Tekscan } \\
\text { (F-Scan } \\
\text { system) }\end{array}$ & RSF & 0.2 & Ajustable & $960 \max$. & $165 \mathrm{~Hz}$ & $10 \mathrm{Kg} / \mathrm{cm}^{2}$ & $\begin{array}{l}4 \text { Sensores/ } \\
\mathrm{cm}^{2} \text { (res. } \\
\text { Espacial) }\end{array}$ & $\begin{array}{l}\text { Alta resolución } \\
\text { espacial. Bajo costo }\end{array}$ \\
\hline $\begin{array}{l}\text { Parotec } \\
\text { System }\end{array}$ & Hidroceldas & 3 & $\begin{array}{c}23 / 24 a \\
47 / 48\end{array}$ & 24 & $\begin{array}{l}250 \mathrm{~Hz}, \\
\text { ajustable }\end{array}$ & $62 \mathrm{~N} / \mathrm{cm}^{2}$ & $2.5 \mathrm{~N} / \mathrm{cm}^{2}$ & $\begin{array}{c}\text { Mide componentes } \\
\text { normales y } \\
\text { tangenciales de } \\
\text { fuerza pero no } \\
\text { diferencia por } \\
\text { separado su } \\
\text { dirección }\end{array}$ \\
\hline $\begin{array}{c}\text { IBV } \\
\text { (BioFoot) }\end{array}$ & $\begin{array}{l}\text { Cerámicas } \\
\text { Piezoléctricas }\end{array}$ & 0,7 & $29 / 46$ & $64 \max$ & $\begin{array}{l}\text { Hasta } 500 \\
\text { Hz }\end{array}$ & -- & $1 \mathrm{~N} / \mathrm{cm}^{2}$ & $\begin{array}{l}\text { Reversibles. } \\
\text { Medición Bipodal. } \\
\text { Transmisor por } \\
\text { telemetría }\end{array}$ \\
\hline
\end{tabular}

\subsection{Sensor de fuerza triaxial piezoeléctrico}

Las condiciones de activación para la operación de una ortesis activa del miembro inferior, que garantice la seguridad y estabilidad pueden ser obtenidas de forma directa conociendo la orientación y magnitud de la fuerza de reacción que ejerce el suelo sobre las articulaciones. Por ejemplo, en el caso de una articulación ortésica de rodilla que se libera o bloquea durante el ciclo de marcha, se establece una condición de seguridad para dicho funcionamiento, dependiendo de la situación instantánea del Vector de Fuerza de Reacción del Suelo (VFRS) con respecto a la articulación, ver figura 3.

En la actualidad los sensores utilizados para la medida de la presión con plantillas instrumentadas están limitados a la medida de fuerzas verticales. La determinación de las componentes cortantes medio-lateral y antero-posterior, son exclusivas de las plataformas de fuerza. Por esto es habitual la combinación de medidas obtenidas con plantillas y plataformas. Pero en el caso de las ortesis del miembro inferior que se conciben para asistir la locomoción a los pacientes con desórdenes en la marcha en actividades cotidianas, es indispensable obtener el VFRS mediante un sistema de medida autónomo y portátil.

\subsubsection{FUNDAMENTOS TEÓRICOS.}

La determinación triaxial de la fuerza de reacción puede ser posible mediante sensores de tipo piezoeléctrico que sean integrables en una plantilla. Para ello es necesario introducir un diseño basado en la respuesta piezoeléctrica.

Debido al efecto piezoeléctrico directo, la aplicación de una tensión mecánica produce la aparición de un campo eléctrico, por lo tanto la aplicación de un esfuerzo mecánico en forma dinámica provocará la generación de una corriente eléctrica. Dependiendo de la disposición de los electrodos y de la dirección de polarización en el elemento piezoeléctrico se obtendrá una señal eléctrica proporcional al esfuerzo en una determinada dirección. El material piezoeléctrico presenta unas ecuaciones constitutivas, en las que se deben considerar los efectos de acoplamiento debidos a las variaciones en la deformación y el campo eléctrico en las tres direcciones ortogonales. La notación de tensores que se adopta y los ejes de referencia son los reflejados en la figura 5 .

Definiendo la dirección de polarización de la cerámica como el eje 
3, se tiene una relación entre la tensión mecánica experimentada por el material, el campo eléctrico aplicado, y el desplazamiento eléctrico resultante, dada por:

$$
D_{i}=\varepsilon_{i} E_{i}+d_{i j} T_{j}
$$

donde $d_{i j}$ es el tensor de constantes piezoeléctricas de carga, habitualmente denominadas simplemente constantes piezoeléctricas, $D_{i}$ es el vector de desplazamiento eléctrico, $\mathrm{E}_{\mathrm{i}}$ es el vector campo eléctrico, $\varepsilon_{\mathrm{ij}}$ es el tensor permeabilidad eléctrica y $\mathrm{T}_{\mathrm{j}}$ es el tensor de esfuerzos mecánicos. Por convención, el primer subíndice de las constantes piezoeléctricas indica la dirección del campo y el segundo la dirección del esfuerzo aplicado. Una cerámica piezoeléctrica tiene sólo un tipo de matriz de constantes piezoeléctricas, sea cual sea la simetría de los monocristales constituyentes. Como todo material policristalino, la cerámica piezoeléctrica es inicialmente isótropa, pero esta isotropía es destruida en la dirección del campo de polarización, manteniéndose en el plano perpendicular a este. Esta isotropía planar de la cerámica piezoeléctrica se expresa en sus constantes piezoeléctricas por las igualdades: $d_{32}=d_{31} y$ $\mathrm{d}_{24}=\mathrm{d}_{15}$. La primera ecuación significa que un desplazamiento eléctrico paralelo al eje de polarización 3 (Z) interactúa de la misma manera con las tensiones mecánicas axiales a lo largo de cualquiera de los ejes $1(\mathrm{X})$ y 2 (Y). La segunda relación establece que un desplazamiento eléctrico paralelo al eje $2(\mathrm{Y})$ interacciona en la misma manera con un esfuerzo de cortadura en el plano 4 (YZ) que un desplazamiento a lo largo del eje $1(X)$ con un esfuerzo de corte en el plano $5(X Z)$.

Se define entonces un tensor piezoeléctrico para una cerámica piezoeléctrica polarizada en el eje $3, \mathrm{Z}$, con la siguiente matriz:

$$
\left[\begin{array}{cccccc}
0 & 0 & 0 & 0 & d_{15} & 0 \\
0 & 0 & 0 & d_{15} & 0 & 0 \\
d_{31} & d_{31} & d_{33} & 0 & 0 & 0
\end{array}\right]
$$

Para esta configuración los valores $\varepsilon_{2}$ y $\varepsilon_{1}$ coinciden para la simetría considerada (cuando la cerámica piezoeléctrica está polarizada según la dirección 3).

Por lo tanto se pueden reemplazar en la ecuación general (1) así:

$$
\begin{aligned}
& D_{1}=\varepsilon_{1} E_{1}+d_{15} T_{5} \\
& D_{2}=\varepsilon_{1} E_{2}+d_{15} T_{4} \\
& D_{3}=\varepsilon_{3} E_{3}+d_{31}\left(T_{1}+T_{2}\right)+d_{33} T_{3}
\end{aligned}
$$

Los parámetros piezoeléctricos de interés al considerar los efectos electromecánicos de los materiales piezoeléctricos son las constantes piezoeléctricas de carga $\left(\mathrm{d}_{31} \mathrm{y} \mathrm{d}_{33}\right)$, las constantes piezoeléctricas de voltaje $\left(\mathrm{g}_{31} \mathrm{y} \mathrm{g}_{33}\right)$ y los factores de acoplamiento electromecánico $\left(\mathrm{k}_{31^{\prime}}\right.$ $\mathrm{k}_{33^{\prime}}, \mathrm{k}_{\mathrm{p}} \mathrm{y} \mathrm{k}_{\mathrm{t}}$.

Las expresiones anteriores definen las ecuaciones constitutivas de la cerámica piezoeléctrica. Cuando sobre la cerámica piezoeléctrica se disponen electrodos con una configuración particular, se están imponiendo superficies equipotenciales que dan lugar a modificaciones en el desplazamiento eléctrico, $\mathrm{D}_{\mathrm{i}^{\prime}}$ puesto de manifiesto bajo la aplicación de esfuerzos mecánicos. Bajo estas condiciones el estudio analítico de las ecuaciones constitutivas carece de sentido y se impone el empleo de métodos numéricos de análisis para evaluar el efecto de los esfuerzos mecánicos aplicados sobre el desplazamiento eléctrico desarrollado. A partir de una cerámica piezoeléctrica polarizada según el eje 3 se estudian los efectos de los electrodos en diferentes configuraciones sobre el elemento, analizando los efectos de acoplamiento y la posibilidad de obtener una medida de las componentes en las tres direcciones de desplazamiento haciendo uso de un solo elemento mediante una medición multiplexada entre electrodos.

$\mathrm{Si}$ se tienen los electrodos como en el elemento mostrado en la figura 6a, se obtiene la componente $\mathrm{D}_{3} \mathrm{y}$ desplazamiento eléctrico (carga eléctrica entre electrodos perpendiculares al eje 3) bajo la aplicación de

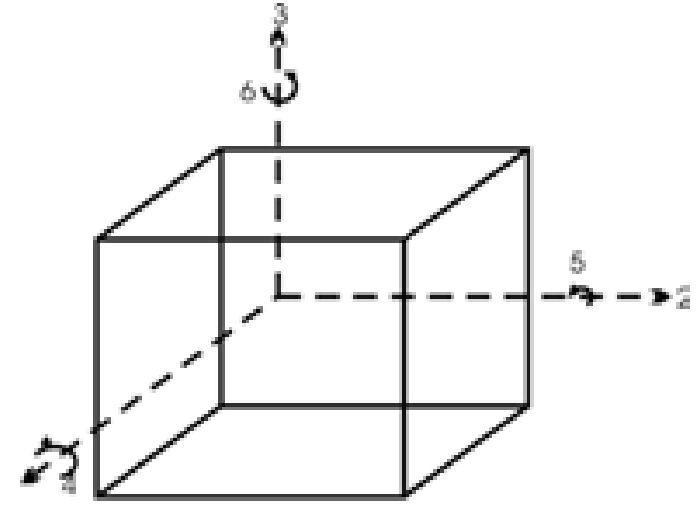

Figura 5. Convención para la expresión de las ecuaciones constitutivas del medio piezoeléctrico.

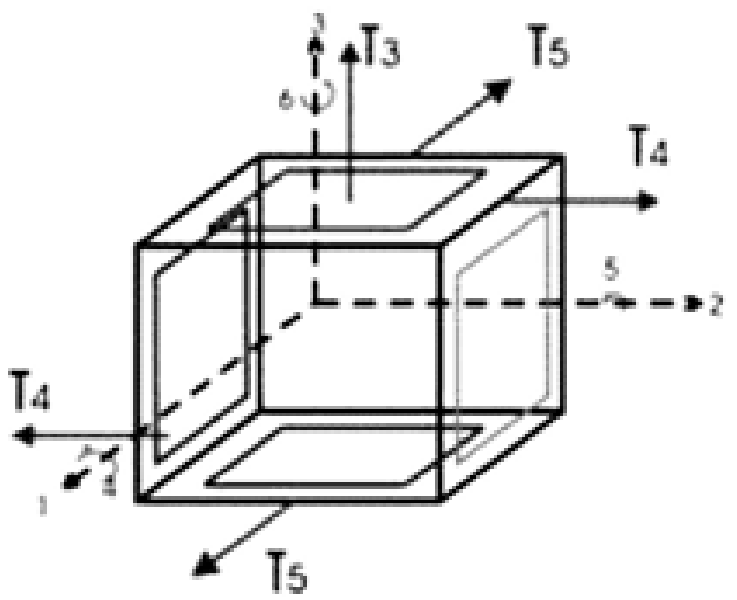

a)

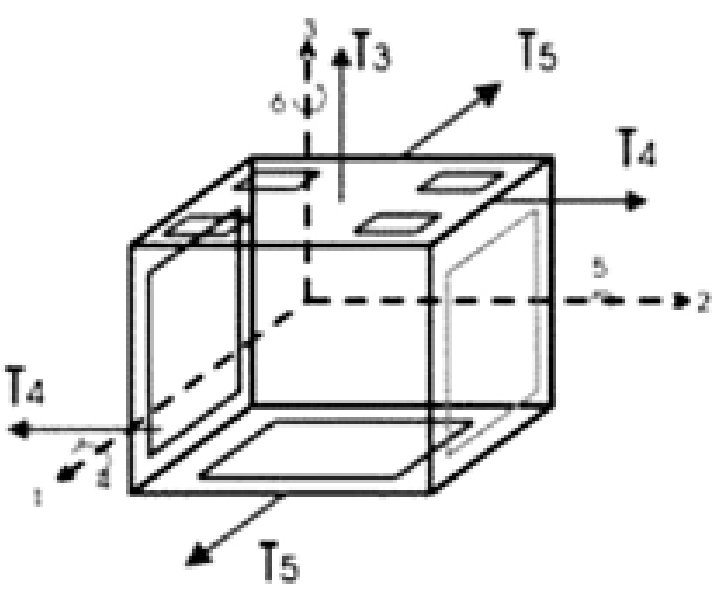

b)

Figura 6. Disposición de los electrodos y los esfuerzos para la medida triaxial de la fuerza de contacto entre el pie y el suelo.

cargas mecánicas normales en la dirección 3. Esta configuración es teóricamente insensible a tensiones de corte según las direcciones 4 y 5 .

De forma similar, con la misma configuración, la componente $\mathrm{D}_{2}$ del desplazamiento eléctrico es proporcional a la carga mecánica de corte según la dirección 4 . De nuevo, teóricamente este desplazamiento eléctrico es independiente de la carga mecánica normal en la dirección 3. 


\subsubsection{SIMULACIÓN MEDIANTE ANÁLISIS POR ELEMENTOS FINITOS.}

Con el fin de comprobar estos resultados teóricos, se ha realizado un estudio por elementos finitos de un sensor triaxial, en el que se supone una polarización de la cerámica según el eje 3 (Z) y en el que se han dispuesto electrodos en las caras perpendiculares a los ejes $1(X)$ y 2 (Y). En el estudio se consideran circuitos de medida independientes para cada uno de los pares de electrodos. Para ello se ha hecho uso del entorno ATILA en el que se ha simulado un caso de carga armónico.

La configuración propuesta es tal que, ante una carga normal en el eje 3 (presión vertical sobre la plantilla), solamente la componente $\mathrm{D}_{3}$ del desplazamiento eléctrico es distinta de cero. Como consecuencia, la tensión eléctrica entre los electrodos perpendiculares al eje 3 es una medida directa de la presión vertical.

Del mismo modo, la componente $\mathrm{D}_{1} \circ \mathrm{D}_{2}$ del desplazamiento eléctrico es únicamente sensible a esfuerzos de cortadura según las direcciones 5 y 4 respectivamente. Como consecuencia, la tensión eléctrica entre electrodos perpendiculares a la dirección 2 es una medida directa de la tensión de cortadura $\mathrm{T}_{4}$. Se ha aplicado una carga según la dirección vertical, véase la figura 7. Como condiciones de contorno, se han especificado superficies equipotenciales en caras perpendiculares a los ejes $2(\mathrm{Y})$ y $3(\mathrm{Z})$. De este modo se modela la presencia de electrodos en estas posiciones, cuyo efecto es el establecer un cortocircuito en toda la superficie.

Se observa en esta figura que existe un gradiente efectivo de potencial a lo largo de la dirección 3, que se traduce en una tensión eléctrica entre los terminales superior e inferior. Es fácilmente observable el efecto de los electrodos laterales que da lugar a un efecto de borde en lo que teóricamente debería ser un campo eléctrico uniforme.

La figura 8 muestra un estudio similar en el que la carga mecánica aplicada es de corte según la dirección 4 . De nuevo se observa que se establece un gradiente de potencial eléctrico entre los electrodos laterales (perpendiculares a la dirección 2). También en esta figura se observa el efecto de borde que introducen los electrodos dispuestos en la dirección 1.

Finalmente, la figura 9 muestra un caso de carga combinada según los ejes 3 y 4 . El estudio demuestra la aparición de gradientes de potencial entre los dos pares de electrodos analizados anteriormente. La
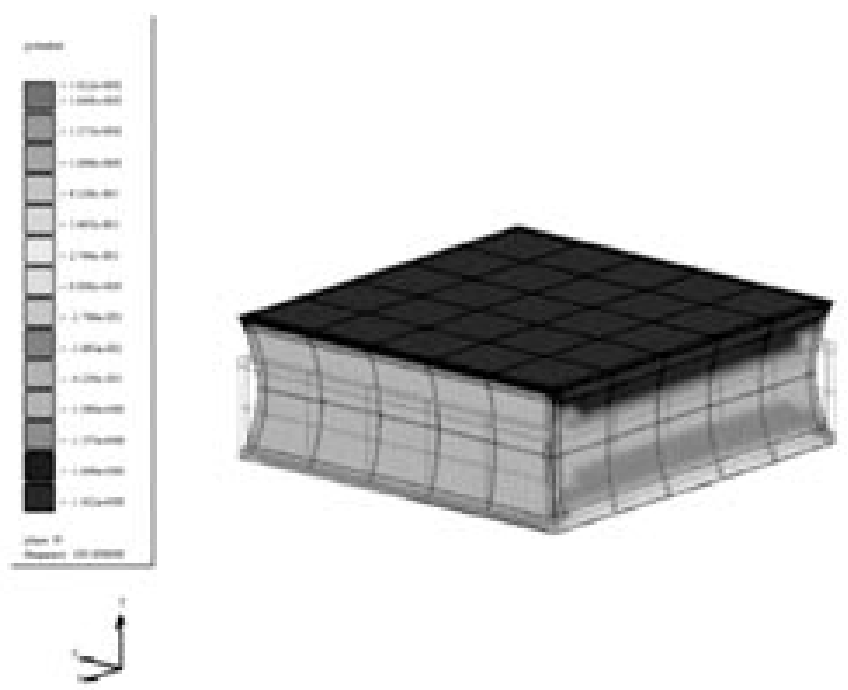

Figura 7. Distribución del potencial eléctrico en el elemento sensor bajo la aplicación de una carga normal en la dirección Z: efecto de la zona cortocircuitada por los electrodos normales a la dirección $Y$ sobre la distribución del campo.

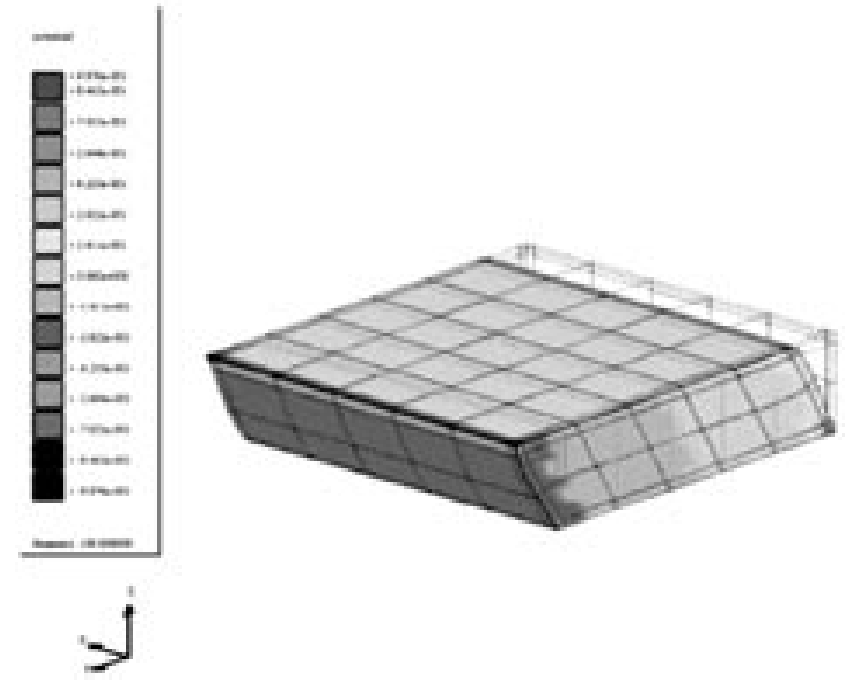

Figura 8. Distribución del potencial eléctrico en el elemento sensor bajo la aplicación de una carga de corte en la dirección Y: efecto de la zona cortocircuitada por los electrodos normales a la dirección $\mathrm{Z}$ sobre la sensibilidad a esfuerzos de corte.

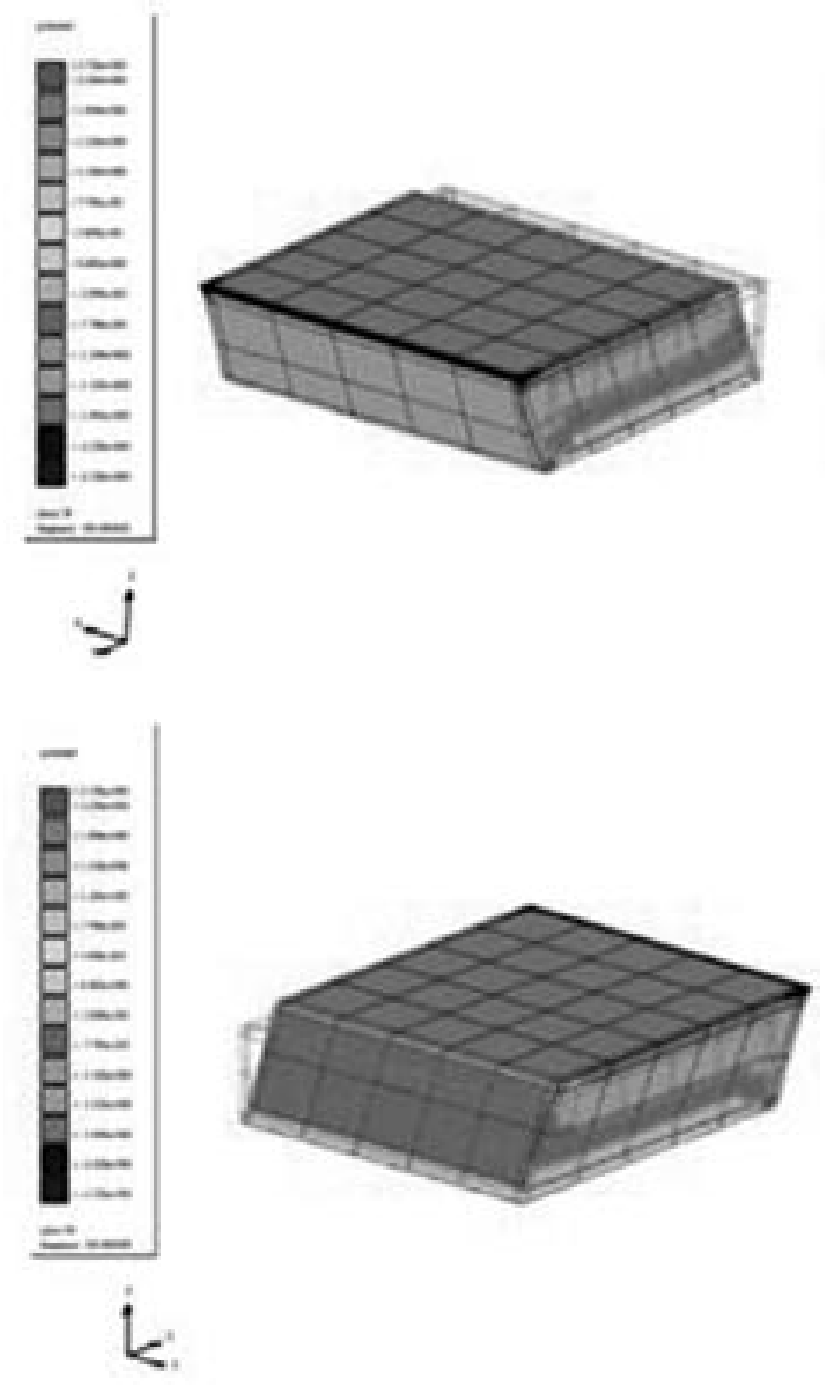

Figura 9. Distribución del potencial eléctrico en el elemento sensor bajo la aplicación combinada de una carga normal en la dirección $\mathrm{Z}$ y una carga de cortadura en la dirección Y: variación bidimensional del potencial eléctrico. 
simulación permite comprobar así la baja sensibilidad cruzada de la configuración propuesta y como consecuencia la posibilidad del empleo de este sensor para la medida concomitante de esfuerzos de corte tipo $\mathrm{T}_{3^{\prime}} \mathrm{y} \mathrm{T}_{4}$. El análisis anterior puede ser completado mediante la adición de un tercer par de electrodos, de forma que la configuración resulte también sensible al esfuerzo $\mathrm{T}_{5}$.

Además de la comprobación experimental en curso, el análisis debe extenderse a otras configuraciones que minimicen la aparición de efectos de borde. En este sentido, se prestará especial atención en el futuro al análisis de configuraciones como la mostrada en la figura $6 \mathrm{~b}$.

\section{CONCLUSIONES}

Se ha introducido el diseño de un sensor triaxial piezoeléctrico para su integración en plantillas para la determinación de presiones plantares durante el ciclo de marcha humana. El elemento sensor piezoeléctrico permite la medida concomitante de los esfuerzos de corte $\mathrm{T}_{3} \mathrm{y} \mathrm{T}_{4}$ debido a la baja sensibilidad cruzada de la configuración propuesta. En el diseño se ha constatado la aparición de gradientes de potencial entre los pares de electrodos analizados en cada caso, y sólo entre ellos.

\section{AGRADECIMIENTOS}

Los autores agradecen el apoyo financiero para la realización de este trabajo bajo el proyecto CICYT-TAP99-1244-CO2-01 (ICARO).

\section{BIBLIOGRAFÍA}

1. P. Ochoa, M. Villegas, J.F. Fernández, Piezocomposites metal cerámica como elementos activos en acelerómetros, Bol. Soc. Esp. Ceram. V., 41[1] 126-130 (2002)

2. J.A. Casals, A. Albareda, R. Pérez, F. Montero de Espinosa, Caracterización no lineal con excitación a ráfagas de transductores de potencia con piezocomposites 1-3 para emisión en aire, Bol. Soc. Esp. Ceram. V., 41[1] 102-106 (2002)

3. X. Jordá, M. Lozano, E. Chico, A. Lozano, H. Amaveda, F. Barrera, Sistema de excitación por pulsos para la caracterización de resonadores para atomización, Bol. Soc. Esp. Ceram. V., 41[1] 85-91(2002)

4. J.L. Pons, H. Rodriguez, I. Luyckx, D. Reynaerts, R. Ceres, H. Van Brussel, High torque ultrasonic motors for hand prosthetics: current status and trends, Tech. Health Care, 10(2): 121-133, (2002)

5. M.J. Warren-Forward, R.M. Goodall, D.J. Pratt, Three-dimensional displacement and force transducer, Meas. Sci. Tech., 139 [1], 21 -29 (1992).

6. J.E. Sanders, L.M. Smith, F.A. Spelman, D.J. Warren, A portable measurement system for prosthetic triaxial force transducers, IEEE T. Rehab., 3 [4], 366 -373 (1995)

7. J.J. Sánchez-Lacuesta et al., Biomecánica de la marcha humana normal y patológica, Publicaciones IBV (1999).

8. P.M. Quesada, G.S. Rash, Simultaneous Pedar and Fscan plantar pressure measurements during walking. Poster presentation: Twenty-First Annual Meeting of the

American Society of Biomechanics Clemson University, South Carolina September 24-27, (1997)

9. L.A. Boyd., E.L. Bontrager, The reliability of the Novel Pedar system of Inshoe pressure measurement during free ambulation. Twenty-First Annual Meeting of the American Society of Biomechanics Clemson University, South Carolina September 24-27, (1997).

10. T.R. Han, N.J. Paik, M. S. Im, Quantification of the path of center of pressure (COP) using an F-scan in-shoe transducer. Gait Posture 10, 284-254 (1999).

11. H.H.C.M. Savelberg, A.L.H. de Lange. Assessment of the horizontal, foreaft component of the ground reaction force from insole pressure patterns by using artificial neural networks. Clin. Biomech. 14, 585-592 (1999)

12. T.G. McPoil, M.N. Orli,. Plantar Pressure Assesment. Phys. Ther., 80 [4], (2000) 